\title{
Overview of W7-X ECRH Results in OP1.2a
}

\author{
H.P. Laqua $^{1}$, J. Baldzuhn ${ }^{1}$, H. Braune ${ }^{1}$, S. Bozhenkov ${ }^{1}$, K.J. Brunner ${ }^{1}$, Ye.O. Kazakov², \\ S.Marsen $^{1}$, D. Moseev ${ }^{1}$, T.Stange ${ }^{1}$, R.C. Wolf ${ }^{1}$, M. Zanini ${ }^{1}$ and Wendelstein7-X Team \\ ${ }^{1}$ Max-Planck-Institute for Plasma Physics, Greifswald, Germany \\ ${ }^{2}$ Laboratory for Plasma Physics, LPP-ERM/KMS, Brussels, Belgium
}

The stellarator Wendelstein7-X was exclusively heated by ECRH in the operation phase OP1.2a. The ECRH system consists of 10 gyrotrons, with an output power of 0.6-1.0 MW each, a quasi optical transmission line and flexible microwave launcher inside the plasma vessel [1]. The over-all transmission efficiency was estimated to be $\sim 94 \%$. The ECRH system is commissioned for $1800 \mathrm{~s}$ operation at full power. It already demonstrated all requirements, which are necessary for a high-performance steady state operation at reactor relevant parameters at W7$\mathrm{X}$. The $140 \mathrm{GHz}$ ECRH uses the second harmonic resonance at $2.5 \mathrm{~T}$ with an absorbed power of up to 7 MW. Besides the reliable plasma start-up und routine ECRH wall conditioning, stationary discharges up to $30 \mathrm{~s}$ have been achieved, which were only limited by the maximum test divertor energy load. Furthermore, the novel remote steering launcher, an important concept for the future fusion reactor, was tested for the first time for plasma heating and current drive. The long discharges were used to demonstrate current control and bootstrap current compensation by ECCD. Localized ECCD strongly changed the rotation transform iota and thus the confinement and stability. In particular by dedicated ECCD strong MHD activity with repetitive central temperature collapse could be driven, which in the worst case lead to a total loss of the plasma confinement. In combination with pellet injection (PI), highest performance with a plasma energy above $1 \mathrm{MJ}$ has been achieved with the X2mode ECRH at a plasmas density of $0.810^{20} \mathrm{~m}^{-3}$ and an ion temperature of $3.8 \mathrm{keV}$, which demonstrates the already good collisional coupling between electrons and ions at that density. Even higher densities have been achieved using the O2-mode combined with PI as shown in Fig.1. Here highperformance plasmas at densities above the X2-cutoff and up to $1.410^{20} \mathrm{~m}^{-3}$ have been demonstrated, which is already close to the envisaged future steady state high-performance plasma scenario. Achieving efficient plasma heating with the $\mathrm{O} 2$ mode requires establishing a sufficiently dense and hot target plasma for the $\mathrm{O} 2$ mode to take over. This was accomplished using the X2-mode for the plasma start-up, and then during the initial two seconds of plasma operation the polarization was changed to $\mathrm{O} 2$ before the start of pellet fuelling. The O2-mode heating efficiency of $>$ $90 \%$ was achieved with the help of individually shaped reflector tiles for the $10 \mathrm{ECRH}$ beams enabling at least 3 passes through the EC-resonance in the plasma center. The reliable and efficient operation was guaranteed by a set of ECRH protective diagnostics, which were specially established for the ECRH requirements. In particular the ECRH stray radiation measurement turned out to be a reliable interlock signal to prohibit ECRH operation with insufficient plasma absorption. Furthermore the position and launch direction of each beam was measured by infrared cameras observing its thermal footprint at the heat shield tiles opposite of the antennas. Finally selected $140 \mathrm{GHz}$ beams was also used as a source for the collective Thomson scattering (CTS), which was also commissioned [2]. Here a local ion temperature measurement was demonstrated (see Fig.2). For the next operational campaign op1.2b new ECRH scenarios have been prepared. In particular the operation at a reduced magnetic field strength of $1.75 \mathrm{~T}$ requires the use of the third harmonic $\mathrm{x}$ mode (X3). Here ray-tracing calculation predicted sufficiently high absorption for plasma build-up and sustain if the electron temperature is above $200 \mathrm{eV}$. The plasma start-up with X3 needs probably an assistance by another heating method, like X2 ECRH at $105 \mathrm{GHz}$ or ICRH. It is also planed to test a new method to excite waves at the ion cyclotron (IC) frequency with ECRH. The ECRH power will be modulated with the IC-frequency using the beat wave of two crossing ECRH beams. For the conversion into IC-oscillation the magnetic field strength variation along the magnetic axis will be used. The electron perpendicular magnetic momentum $\mathbf{M}$ will be increased by the EC-interaction and the grad $\mathbf{B} \cdot \mathbf{M}$ force will drive the oscillation.

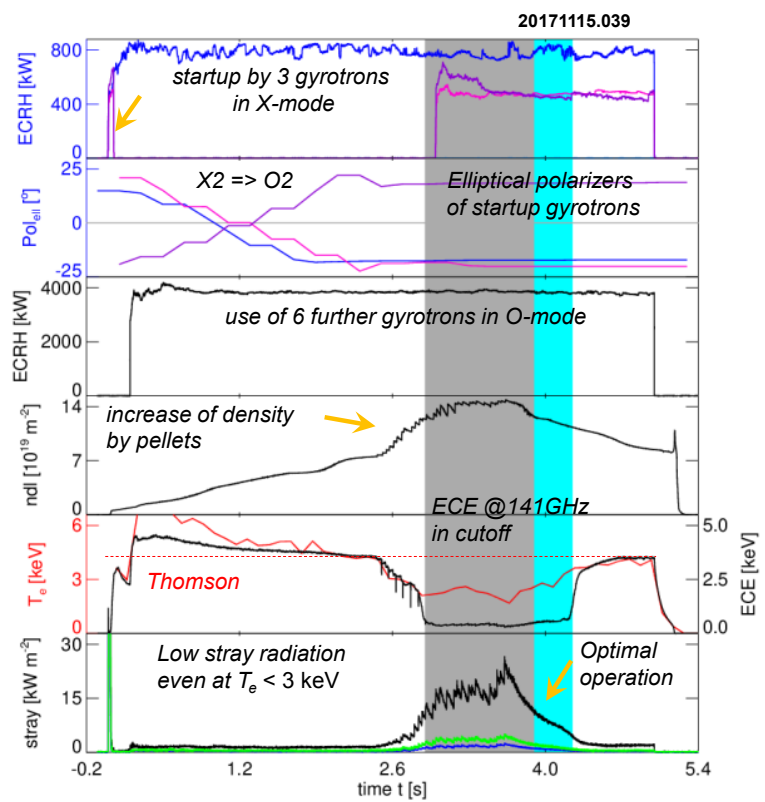


Fig. 1. High density plasma scenario with O2-ECRH and pellet injection. The second row shows the chance of polarizer position for the 3 "start-up" gyrotron beams, when their polarisation was switched from X2 to $\mathrm{O} 2$.

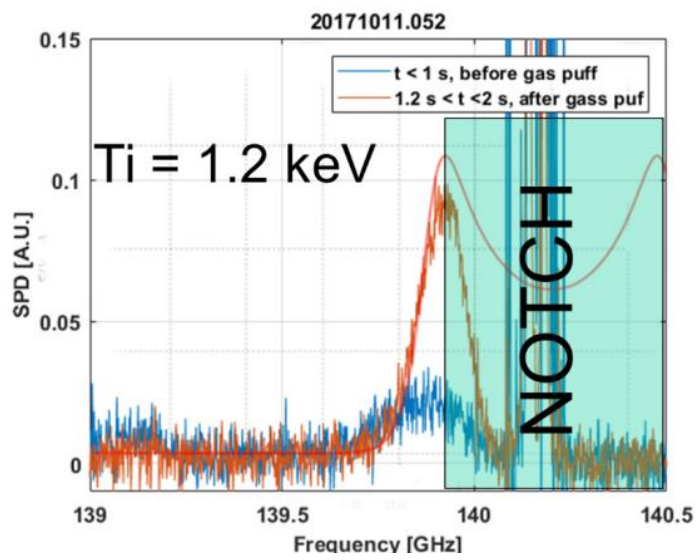

Fig. 2. CTS spectrum at low density (bleu) and medium density (red) in a Helium plasma.

1. Erckmann V. et al.(2007) Electron Cyclotron Heating for W7-X: Physics and Technology, Fusion Science and Technology, 52:2, 291-312, DOI: 10.13182/FST07-A1508

2. Moseev D. et al. Collective Thomson Scattering Diagnostic at Wendelstein 7-X submitted to review of scientific instruments 